\title{
Changes in LDL-C levels and goal attainment associated with addition of ezetimibe to simvastatin, atorvastatin, or rosuvastatin compared with titrating statin monotherapy
}

\author{
This article was published in the following Dove Press journal: \\ Vascular Health and Risk Management \\ 14 November 2013 \\ Number of times this article has been viewed
}

\author{
JoAnne M Foody' \\ Peter P Toth ${ }^{2,3}$ \\ Joanne ETomassini ${ }^{4}$ \\ Shiva Sajjan ${ }^{4}$ \\ Dena R Ramey ${ }^{4}$ \\ David Neff ${ }^{4}$ \\ Andrew M Tershakovec ${ }^{4}$ \\ Henry $\mathrm{Hu}^{4}$ \\ Kaan Tunceli ${ }^{4}$ \\ 'Brigham and Women's Hospital, \\ Boston, MA, ${ }^{2} \mathrm{CGH}$ Medical Center, \\ Sterling, IL, ${ }^{3}$ University of Illinois \\ College of Medicine, Peoria, IL, ${ }^{4}$ Merck \\ \& Co., Inc., Whitehouse Station, NJ, \\ USA
}

\begin{abstract}
Background: Many high-risk coronary heart disease (CHD) patients on statin monotherapy do not achieve guideline-recommended low-density lipoprotein cholesterol (LDL-C) goals, and combination lipid-lowering therapy may be considered for these individuals. The effect of adding ezetimibe to simvastatin, atorvastatin, or rosuvastatin therapy versus titrating these statins on LDL-C changes and goal attainment in CHD or CHD risk-equivalent patients was assessed in a large, managed-care database in the US.
\end{abstract}

Methods: Eligible patients $(\mathrm{n}=17,830)$, initially on statin monotherapy who were $\geq 18$ years with baseline and follow-up LDL-C values, no concomitant use of other lipid-lowering therapy, and on lipid-lowering therapy for $\geq 42$ days, were identified between November 1, 2002 and September 30, 2009. The percent change from baseline in LDL-C levels and the odds ratios for attainment of LDL-C $<1.8$ and $<2.6 \mathrm{mmol} / \mathrm{L}$ (70 and $100 \mathrm{mg} / \mathrm{dL}$ ) were estimated using an analysis of covariance and logistic regression, respectively, adjusted for various baseline factors.

Results: LDL-C reductions from baseline and goal attainment improved substantially in patients treated with ezetimibe added onto simvastatin, atorvastatin, or rosuvastatin therapy $(\mathrm{n}=2,312)$ versus those $(\mathrm{n}=13,053)$ who titrated these statins. In multivariable models, percent change from baseline in LDL-C was $-13.1 \%$ to $-14.8 \%$ greater for those who added ezetimibe onto simvastatin, atorvastatin, or rosuvastatin versus those who titrated. The odds of attaining LDL-C $<1.8$ and $<2.6 \mathrm{mmol} / \mathrm{L}$ ( 70 and $100 \mathrm{mg} / \mathrm{dL}$ ) increased by 2.6-3.2-fold and 2.5-3.1-fold, respectively, in patients who added ezetimibe onto simvastatin, atorvastatin, or rosuvastatin versus titrating statins.

Conclusion: $\mathrm{CHD} / \mathrm{CHD}$ risk-equivalent patients in a large US managed-care database, who added ezetimibe onto simvastatin, atorvastatin, or rosuvastatin, had greater LDL-C reductions and goal attainment than those who uptitrated these statin therapies. Our study suggests that high-risk CHD patients in need of more intensive LDL-C lowering therapy may benefit by adding ezetimibe onto statin therapy.

Keywords: low-density lipoprotein cholesterol goal, ezetimibe, atorvastatin, rosuvastatin

\section{Introduction}

While statin therapy has been shown to be highly effective in lowering low-density lipoprotein cholesterol (LDL-C) and reducing cardiovascular disease risk, ${ }^{1-3}$ many higher-risk coronary heart disease (CHD) and/or CHD risk-equivalent patients do not achieve their guideline-recommended LDL-C goals. ${ }^{4,5}$ Several studies have shown that while approximately two thirds of high-risk CHD patients achieve LDL-C
Correspondence: JoAnne M Foody Brigham and Women's Hospital, I I 53 Centre Street, Suite 4930, Boston, MA 02130, USA

$\mathrm{Tel}+$ I 8573071989

Fax + I 6179832488

Email jfoody@partners.org 
levels of $<2.6 \mathrm{mmol} / \mathrm{L}(100 \mathrm{mg} / \mathrm{dL})$, only about one third of these patients attain LDL-C $<1.8 \mathrm{mmol} / \mathrm{L}(70 \mathrm{mg} / \mathrm{dL})$ levels. ${ }^{4,6-9}$ For these patients, clinical guidelines recommend more intensive LDL-C-lowering therapy, including statin uptitration to maximally tolerated doses, and/or combination therapy. ${ }^{2,3}$ However, many patients may not be able to tolerate higher statin doses, and combination therapy may be a better alternative. In several clinical studies, the addition of ezetimibe to ongoing statin therapy or coadministered with statins has been shown to improve lowering of LDL-C as well as goal attainment more than statins alone and is generally well tolerated in various patient populations. ${ }^{10}$ The combination also provides greater LDL-C reduction and goal attainment when compared with doubling the statin dose. In this study, the effect of adding ezetimibe to simvastatin, atorvastatin, or rosuvastatin therapy on LDL-C changes and goal attainment was compared with titrating these statins in $\mathrm{CHD} / \mathrm{CHD}$ risk-equivalent patients using data from a large US managed-care database.

\section{Materials and methods}

This was a retrospective, observational study of data in a US administrative managed-care database that included medical claims, automated laboratory data, and prescription claims. Eligible patients, identified between November 1, 2002 and September 30, 2009, included those $\geq 18$ years of age who had a prescription for statin monotherapy with baseline and follow-up LDL-C values, as well as no overlap with other lipid-lowering therapy and who had no discontinuations of lipid-lowering therapy at baseline or follow-up during the study period. Eligibility included a diagnosis of CHD or CHD risk-equivalent in the 12 months prior to the switch to ezetimibe add-on therapy or titrating statin therapy. Diagnoses (derived from the Ninth Revision of International Classification of Diseases or inferred from Current Procedural Terminology procedure codes) considered to be $\mathrm{CHD}$ and CHD risk-equivalents included myocardial infarction, angina pectoris, ischemic stroke, peripheral vascular disease, diabetes, other acute/subacute ischemic heart disease, coronary or peripheral revascularization, acute carotid artery procedures, other forms of chronic ischemic heart disease, and atherosclerosis. Although a 10-year Framingham risk score that exceeds $20 \%$ is also a CHD risk-equivalent, information to calculate Framingham risk score was not available in this database.

The add-on group consisted of those patients who were initially on simvastatin, atorvastatin, or rosuvastatin monotherapy and added ezetimibe onto this statin therapy. The titrator group consisted of those patients who either titrated their initial statin dose or switched to higher-potency statin monotherapy. The comparative potency levels for the various doses of simvastatin, atorvastatin, or rosuvastatin, and for ezetimibe added onto each of these statins, was estimated from the expected LDL-C responses based on the prescribing information and clinical trial data for the indicated therapies (Table 1). ${ }^{11-24}$ The potency levels based on estimated LDLC-lowering reductions were categorized as low $(20 \%-32 \%)$, medium $(36 \%-50 \%)$, or high $(52 \%-70 \%)$. Baseline was defined as up to 6 months before the add-on or titration date (index date), with the last LDL-C value taken after at least

Table I Estimated low-density lipoprotein cholesterol (LDL-C) lowering efficacy levels for statin and ezetimibe added onto statin therapies ${ }^{\mathrm{a}}$

\begin{tabular}{|c|c|c|c|c|c|c|c|}
\hline \multirow{2}{*}{$\begin{array}{l}\text { LDL-C- } \\
\text { lowering } \\
\text { potency level* }\end{array}$} & \multirow{2}{*}{$\begin{array}{l}\text { Estimated \% } \\
\text { LDL-C reduction } \\
\text { range** }\end{array}$} & \multicolumn{6}{|c|}{ Mg (estimated \% LDL-C reduction) } \\
\hline & & Rosuvastatin $^{(\mathbf{b})}$ & Atorvastatin $^{(c)}$ & Simvastatin ${ }^{(d)}$ & $\begin{array}{l}\text { Ezetimibe }+ \\
\text { rosuvastatin }\end{array}$ & $\begin{array}{l}\text { Ezetimibe }+ \\
\text { atorvastatin }^{(\mathrm{e})}\end{array}$ & $\begin{array}{l}\text { Ezetimibe }+ \\
\text { simvastatin }\end{array}$ \\
\hline \multirow[t]{2}{*}{ Low } & $20-32$ & & & $5(26)$ & & & \\
\hline & & & & $10(32)^{\dagger}$ & & & \\
\hline \multirow[t]{3}{*}{ Medium } & $36-50$ & & $10(38)$ & $20(36)^{\dagger}$ & & & \\
\hline & & $5(45)$ & $20(44)$ & $40(4 I)^{\dagger}$ & & & \\
\hline & & $10(49)^{\ddagger}$ & $40(50)$ & & & & $10(47)^{\S}$ \\
\hline \multirow[t]{5}{*}{ High } & $52-70$ & $20(54)^{\ddagger}$ & $80(55)$ & & & $10(53)$ & $20(52)^{\S}$ \\
\hline & & $40(60)^{\ddagger}$ & & & $5(59)^{\pi}$ & $20(54)$ & $40(56)^{\S}$ \\
\hline & & & & & $10(61)^{t \dagger}$ & $40(56)$ & $80(60) \|$ \\
\hline & & & & & $20(66)^{\pi}$ & $80(6 I)$ & \\
\hline & & & & & $40(70)^{\ddagger \ddagger}$ & & \\
\hline
\end{tabular}

Notes: ${ }^{a}$ Reprinted with modification from Toth PP, Ballantyne CM, Davidson MH et al. Changes in prescription patterns before and after reporting of the Ezetimibe and Simvastatin in Hypercholesterolemia Enhances Atherosclerosis Regression trial (ENHANCE) results and expected effects on low-density lipoprotein-cholesterol reduction. J Clin Lipidol. 2012;6(2): 180-191. Copyright 2012, with permission from Elsevier." *Potency levels low, medium, and high based on LDL-C-lowering as described in prescribing information for each drug and in previously reported randomized clinical trials as indicated; **data are not placebo-adjusted. \% LDL-C reductions from: ${ }^{\text {b }} \mathrm{Crestor}$ prescribing information; ${ }^{12}$ average of data in Lipitor prescribing information ${ }^{13}$ and clinical studies; ${ }^{17-19}$ 'Zocor prescribing information; ${ }^{14}$ eLiptruzet prescribing information; ${ }^{15}$ †average of data in Zocor prescribing information ${ }^{14}$ and Bays et al; ${ }^{20}$ faverage of data in Crestor prescribing information ${ }^{12}$ and Catapano et al; ${ }^{21}$ §average of data in Vytorin prescribing information ${ }^{16}$ and Ballantyne et al; ${ }^{17,18}$ | Tcalculated using 26\% LDL-C reduction in Pearson, et al; ${ }^{22} \|$ average of data in Vytorin prescribing information, ${ }^{16}$ Ballantyne, et al. ${ }^{17,18}$ and Catapano et al; ${ }^{21} \dagger \nmid$ data from Kosoglou et al $;{ }^{23} \neq \ddagger$ data from Ballantyne et al. ${ }^{24}$ 
42 days on therapy. Follow-up was defined as up to 12 months after the index date, with the first LDL-C value taken after at least 42 days on add-on/titration therapy. A 42-day (6-week) window was selected as the minimum treatment duration prior to an LDL-C value for initial therapy and add-on/titration therapy. This time period was chosen since clinical trials for lipid-lowering agents typically have a minimum 6-week follow-up period to assess LDL-C-lowering efficacy.

The mean percent change from baseline in LDL-C and percentage of patients attaining LDL-C goals $<1.8 \mathrm{mmol} / \mathrm{L}$ $(70 \mathrm{mg} / \mathrm{dL})$ and $<2.6 \mathrm{mmol} / \mathrm{L}(100 \mathrm{mg} / \mathrm{dL})$ were evaluated. The percent change from baseline in LDL-C was assessed using an analysis of covariance model adjusted for age, sex, baseline LDL-C, and baseline statin potency. The odds ratios for the attainment of LDL-C levels $<1.8$ and $<2.6 \mathrm{mmol} / \mathrm{L}$ (70 and $100 \mathrm{mg} / \mathrm{dL}$ ) at follow-up for each baseline therapy were estimated by logistic regression analysis adjusted for age, sex, baseline goal attainment, and baseline statin potency.

\section{Results}

There were 436,370 patients identified in the database between November 1, 2002 and September 30, 2009 who initially received statin monotherapy (Figure 1). Of the $43,492(10 \%)$ patients who added ezetimibe to their ongoing statin therapy, there were $2,312(5.3 \%) \mathrm{CHD} / \mathrm{CHD}$ riskequivalent patients who were eligible for the analysis. This group included $540(23.3 \%)$ patients who were on ongoing simvastatin, 1,504 (65.0\%) on atorvastatin, and $268(11.6 \%)$ on rosuvastatin monotherapies. Of the 392,878 patients $(90 \%)$ in the database initially on statin therapy who titrated to either a higher dose of the same statin or switched to a higher-potency dose of another statin, 13,053 (3.3\%) were eligible for the analysis. Among these patients, 4,170 (32.0\%) were on simvastatin, 7,653 (58.6\%) were on atorvastatin, and $1,230(9.4 \%)$ were on rosuvastatin monotherapies.

The mean age ranged from 56.2 to 57.1 years across the groups and men accounted for about $65.0 \%$ of the overall analysis population (Table 2). The most prevalent diagnoses were diabetes $(44.0 \%-69.0 \%)$ and chronic ischemic heart disease $(32.6 \%-63.1 \%)$. At baseline, the largest proportion of patients on simvastatin monotherapy were on a $40 \mathrm{mg}$ dose in the ezetimibe add-on group, and most of those in the titrator group were on a $20 \mathrm{mg}$ dose. Among the patients on atorvastatin, the greatest proportion of patients were on a $40 \mathrm{mg}$ dose in the add-on group and a $10 \mathrm{mg}$ dose in the titrator group, while among those on rosuvastatin, more patients were on a $10 \mathrm{mg}$ dose in both the add-on and titrator groups. At follow-up, the majority of patients in the simvastatin and atorvastatin add-on groups were still at the baseline dose, while in the rosuvastatin add-on group there was a slight increase in the number of patients who uptitrated to the next dose. Most patients who titrated doubled their doses in each statin group.

The majority of patients were on statins of medium LDL-C-lowering potency at baseline in all groups. Most of the patients on simvastatin monotherapy were on doses

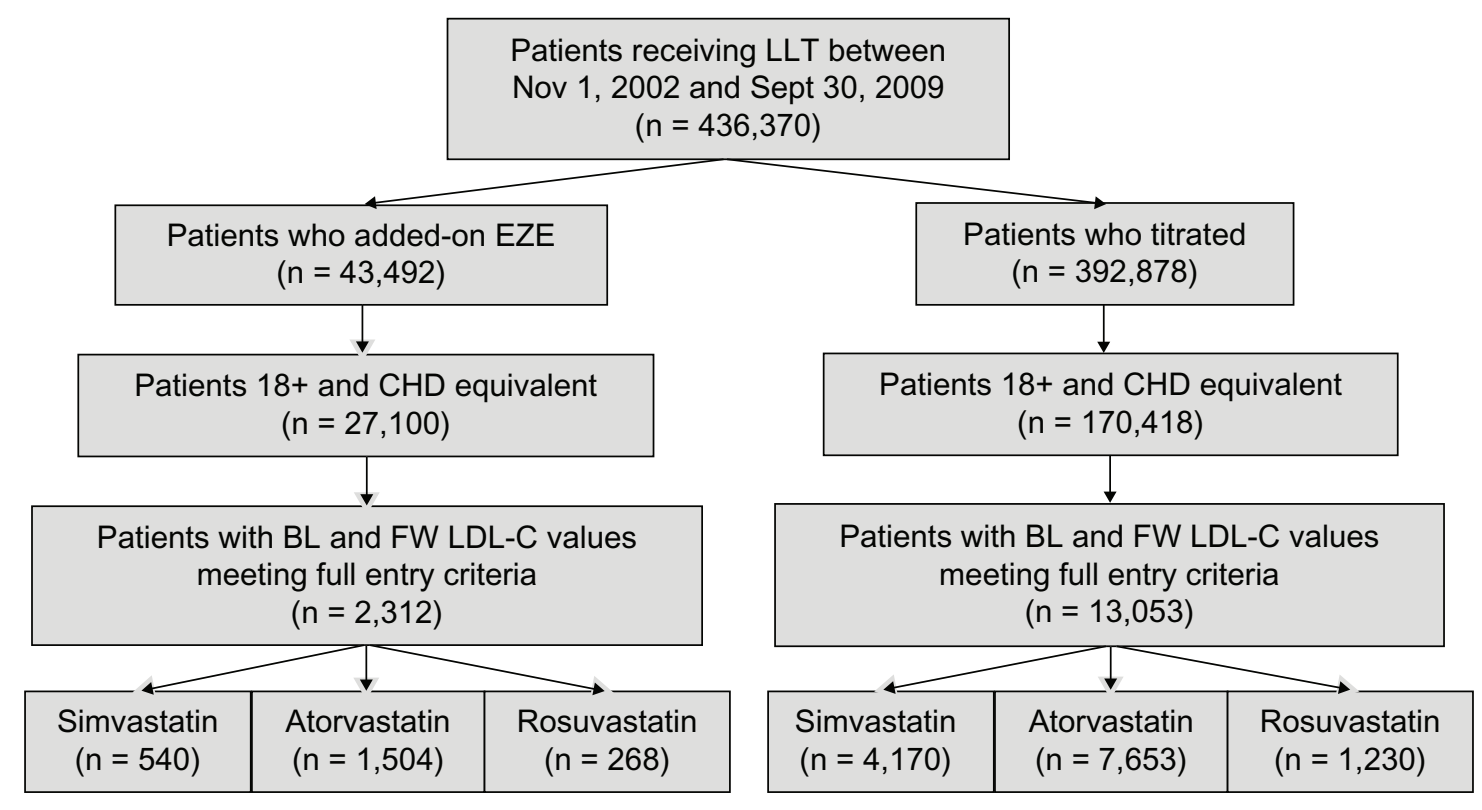

Figure I Patient flow.

Abbreviations: BL, baseline; EZE, ezetimibe; FW, follow-up; LLT, lipid-lowering therapy; CHD, coronary heart disease; LDL-C, low-density lipoprotein cholesterol. 
Table 2 Baseline patient characteristics

\begin{tabular}{|c|c|c|c|c|c|c|}
\hline \multirow[t]{2}{*}{ Characteristic } & \multicolumn{2}{|l|}{ Simvastatin } & \multicolumn{2}{|l|}{ Atorvastatin } & \multicolumn{2}{|l|}{ Rosuvastatin } \\
\hline & $\begin{array}{l}\text { Add-on } \\
(n=540)\end{array}$ & $\begin{array}{l}\text { Titrate } \\
(n=4,170)\end{array}$ & $\begin{array}{l}\text { Add-on } \\
(n=1,504)\end{array}$ & $\begin{array}{l}\text { Titrate } \\
(n=7,653)\end{array}$ & $\begin{array}{l}\text { Add-on } \\
(n=268)\end{array}$ & $\begin{array}{l}\text { Titrate } \\
(\mathrm{n}=1,230)\end{array}$ \\
\hline Age, years, mean (SD) & $57.1(7.9)$ & $56.2(8.1)$ & $57.2(7.4)$ & $56.3(8.2)$ & $56.5(8.3)$ & $56.2(8.1)$ \\
\hline Sex, males, $\%$ & 67.8 & 58.7 & 71.3 & 62.1 & 68.3 & 61.7 \\
\hline Time to index date, ${ }^{*}$ mean days (SD) & $525.0(399.8)$ & $460.7(4 \mid 7.2)$ & $617.0(484.8)$ & $54 I .2(464.3)$ & 355.7 (278.7) & $322.4(255.5)$ \\
\hline $\begin{array}{l}\text { Days to baseline LDL-C } \\
\text { (from initial start), mean (SD) }\end{array}$ & $495.7(396.4)$ & $421.0(4 \mid 3.3)$ & $583.0(485.2)$ & $496.9(46 \mid .2)$ & $324.1(274.9)$ & $280.2(249.5)$ \\
\hline $\begin{array}{l}\text { Days to follow-up LDL-C } \\
\text { (from index date), mean (SD) }\end{array}$ & I $70.8(94.9)$ & $191.4(96.1)$ & $192.7(97.1)$ & $198.7(96.1)$ & $176.6(94.3)$ & $184.0(94.4)$ \\
\hline \multicolumn{7}{|l|}{ Year of index date \% } \\
\hline 2002 & 3.0 & 0 & I.I & 0 & 0 & 0 \\
\hline 2003 & 32.2 & 11.8 & 17.6 & 10.1 & 0 & 0 \\
\hline 2004 & 34.4 & 16.5 & 22.5 & 17.7 & 12.7 & 6.7 \\
\hline 2005 & II.I & 12.7 & 25.6 & 22.7 & 23.1 & $1 \mathrm{I} .4$ \\
\hline 2006 & 7.4 & 14.0 & 19.4 & 20.3 & 20.9 & 17.6 \\
\hline 2007 & 4.4 & 12.0 & 8.6 & 12.0 & 20.1 & 17.6 \\
\hline 2008 & 5.0 & 20.7 & 3.3 & 11.4 & 12.7 & 30.2 \\
\hline 2009 & 2.4 & 12.3 & 1.9 & 5.7 & 10.4 & 16.7 \\
\hline \multicolumn{7}{|l|}{ Baseline comorbidities \% } \\
\hline Acute myocardial infarction & 6.5 & 4.4 & 6.9 & 4.6 & 6.7 & 4.4 \\
\hline Previous myocardial infarction & 7.4 & 5.1 & 10.4 & 4.7 & 9.0 & 4.1 \\
\hline Ischemic stroke & 2.2 & 1.9 & 2.5 & 2.2 & 0.7 & 2.1 \\
\hline Peripheral vascular disease & 2.6 & 2.0 & 2.8 & 2.0 & 2.2 & 1.3 \\
\hline Diabetes & 53.9 & 69 & 49.3 & 66.9 & 44 & 67.5 \\
\hline Angina pectoris & 17.8 & 9.2 & 14.2 & 9.8 & 15.3 & 10.9 \\
\hline Acute ischemic heart disease & 10.0 & 7.0 & 12.5 & 6.9 & 10.1 & 6.1 \\
\hline Revascularization & 8.0 & 4.7 & 7.9 & 5.2 & 8.6 & 6.3 \\
\hline Acute carotid artery procedures & 0.6 & 0.3 & 0.5 & 0.2 & 0 & 0.4 \\
\hline Chronic ischemic heart disease & 58.9 & 32.6 & 63.1 & 37.7 & 60.1 & 32.5 \\
\hline Atherosclerosis & 22.8 & 15.9 & 23.3 & 17.0 & 25.0 & 18.7 \\
\hline $\mathrm{CHD} / \mathrm{CHD}$ risk-equivalent & 100 & 100 & 100 & 100 & 100 & 100 \\
\hline \multicolumn{7}{|l|}{$\begin{array}{l}\text { Baseline dose } \\
\text { (immediately before LDL-C) \% }\end{array}$} \\
\hline $5 \mathrm{mg}$ & 0.7 & 1.5 & 0 & 0 & 3.4 & 19.9 \\
\hline $10 \mathrm{mg}$ & 2.0 & 15.0 & 9.4 & 51.3 & 43.7 & 62.2 \\
\hline $20 \mathrm{mg}$ & 19.6 & 54.3 & 25.1 & 35.6 & 34 & 17.5 \\
\hline $40 \mathrm{mg}$ & 43.9 & 28.6 & 39.4 & 12.7 & 19 & 0.4 \\
\hline $80 \mathrm{mg}$ & 33.7 & 0.6 & 26.1 & 0.4 & 0 & 0 \\
\hline \multicolumn{7}{|l|}{$\begin{array}{l}\text { Follow-up dose } \\
\text { (immediately before LDL-C) \% }\end{array}$} \\
\hline $5 \mathrm{mg}$ & 0.4 & 0.1 & 0 & 0 & 3.4 & 0.9 \\
\hline $10 \mathrm{mg}$ & 3.3 & 1.9 & 7.8 & 2.7 & 32.8 & 21.1 \\
\hline $20 \mathrm{mg}$ & 16.1 & 14.9 & 22.3 & 44.2 & 38.8 & 56.8 \\
\hline $40 \mathrm{mg}$ & 45.4 & 53.8 & 39.4 & 38.4 & 25 & 21.2 \\
\hline $80 \mathrm{mg}$ & 34.8 & 29.3 & 30.4 & 14.8 & 0 & 0 \\
\hline
\end{tabular}

Note: *Time to index date (non-add-on and non-titrate episodes were applied an index date based on distribution of add-on/titrate).

Abbreviations: CHD, coronary heart disease; LDL-C, low-density lipoprotein cholesterol; SD, standard deviation.

of medium LDL-C-lowering potency in each of the add-on (97.2\%) and titrator (83.5\%) groups at baseline (Tables 1 and 2). Among those on atorvastatin, $73.9 \%$ were on mediumpotency LDL-C-lowering doses and $26.1 \%$ were on highpotency doses in the add-on group, while $99.6 \%$ of patients were on medium-potency doses in the titrator group. For those on rosuvastatin, about half of the patients were on mediumpotency doses and half were on high-potency doses in the add-on group, while in the titrator group, most (82.1\%) were on medium-potency and $17.9 \%$ were on high-potency LDLC-lowering doses. At follow-up, among the patients who added ezetimibe to their statin therapies, the majority (96.3\%) of those on simvastatin and all patients on atorvastatin and rosuvastatin monotherapies were on doses of high-potency LDL-C-lowering therapy (Tables 1 and 2). Among those who uptitrated simvastatin, most (98\%) were on medium-potency LDL-C-lowering doses, and among those who uptitrated atorvastatin, $85.3 \%$ were on medium-potency doses and $14.8 \%$ 
were at high-potency levels at follow-up. All patients were on high LDL-C-lowering potency levels of therapy in both the rosuvastatin add-on and titration groups.

The mean LDL-C levels at baseline were significantly higher in the add-on groups for each statin (2.9-3.1 mmol/L [113-118 mg/dL]) compared with those of the titrators (2.6-2.8 mmol/L [102-108 mg/dL], Table 3). At follow-up, LDL-C levels were reduced more in the add-on groups $(2.1-2.2 \mathrm{mmol} / \mathrm{L}[80-85 \mathrm{mg} / \mathrm{dL}])$ than in the titrator groups (2.3-2.5 mmol/L [87-95 mg/dL]). Both the absolute changes in LDL-C levels (Table 3) and the percent changes from baseline (Figure 2) were significantly greater in the add-on groups than in the titrator groups.

At baseline, the percentages of patients who were at LDL-C levels of $<1.8$ and $<2.6 \mathrm{mmol} / \mathrm{L}$ ( 70 and $100 \mathrm{mg} / \mathrm{dL}$ ) were significantly higher in the titrator than add-on groups for all statins (Figure 3). In contrast, at follow-up, the percentage of goal attainment was significantly larger when ezetimibe was added on for each statin group compared with the titrators. In multivariate models, the adjusted difference in percent change from baseline was greater for the ezetimibe add-on group versus the statin titrator group, and the likelihood of goal attainment was 2.5-3.2 times higher for add-on compared with uptitration therapy (Table 4).

\section{Discussion}

In this real-world setting, both LDL-C lowering and goal attainment improved substantially in $\mathrm{CHD} / \mathrm{CHD}$ risk-equivalent patients who added ezetimibe onto ongoing simvastatin, atorvastatin, or rosuvastatin monotherapies compared with those who uptitrated their statin dose or switched to more potent statin therapy. Regardless of several baseline factors, including age, sex, LDL-C levels, goal attainment, and statin potency, LDL-C reductions and the likelihood of attaining LDL-C $<1.8$ and $<2.6 \mathrm{mmol} / \mathrm{L}$ (70 and $100 \mathrm{mg} / \mathrm{dL}$ ) were greater for those patients who added ezetimibe onto simvastatin, atorvastatin, or rosuvastatin compared with those who uptitrated these statins or switched to more potent statin therapy. LDL-C lowering and goal attainment also improved when statin therapy was uptitrated to more efficacious LDL-C-lowering doses. Overall, these results indicate that high-risk $\mathrm{CHD}$ patients in need of more intensive LDL-C-lowering therapy may benefit from therapies with more potent LDL-C-lowering efficacy, including ezetimibe added onto statins or statin uptitration therapies.

Although LDL-C goal attainment rates have recently improved overall through an increased awareness and use of lipid-lowering therapy, many high-risk CHD patients still do not meet guideline-recommended LDL-C goals on statin monotherapy. ${ }^{4,6,25,26}$ In our study, $22 \%-51 \%$ and $3 \%-15 \%$ of high-risk CHD patients had LDL-C levels of $<2.6$ and $<1.8 \mathrm{mmol} / \mathrm{L}(100$ and $70 \mathrm{mg} / \mathrm{dL})$ at baseline, the majority of whom were on medium-potency doses of simvastatin, atorvastatin, or rosuvastatin. Attainment of LDL-C levels $<2.6 \mathrm{mmol} / \mathrm{L}(100 \mathrm{mg} / \mathrm{dL})$ improved substantially in all patients to $77 \%-84 \%$ with addition of ezetimibe to statin therapy and to $62 \%-70 \%$ with statin uptitration. However, while attainment rates for LDL-C $<1.8 \mathrm{mmol} / \mathrm{L}(70 \mathrm{mg} / \mathrm{dL})$ also increased by $30 \%-36 \%$ with ezetimibe add-on therapy, and by $14 \%-26 \%$ for statin uptitration, approximately two thirds of patients still did not reach these levels, even when high-potency LDL-C lowering efficacy levels of these therapies were used. Overall, these results are consistent with prior studies in various high-risk CHD patients, in which lower percentages of patients attained LDL-C levels $<1.8 \mathrm{mmol} / \mathrm{L}$ $(70 \mathrm{mg} / \mathrm{dL})(6 \%-30 \%)$ than LDL-C levels of $<2.6 \mathrm{mmol} / \mathrm{L}$ (100 mg/dL) (18\%-81\%) with lipid-lowering therapy.,6-9,25

Guidelines recommend that more intensive therapy, such as statin titration and/or combination therapy, may be needed for patients who are not at LDL-C goal. ${ }^{2,3}$ While statins remain the first-line therapy for LDL-C reduction

Table 3 Baseline and follow-up lipid values

\begin{tabular}{|c|c|c|c|c|c|c|}
\hline & \multicolumn{2}{|l|}{ Simvastatin } & \multicolumn{2}{|l|}{ Atorvastatin } & \multicolumn{2}{|l|}{ Rosuvastatin } \\
\hline & $\begin{array}{l}\text { Add-on } \\
(n=540)\end{array}$ & $\begin{array}{l}\text { Titrate } \\
(n=4,170)\end{array}$ & $\begin{array}{l}\text { Add-on } \\
(n=1,504)\end{array}$ & $\begin{array}{l}\text { Titrate } \\
(n=7,653)\end{array}$ & $\begin{array}{l}\text { Add-on } \\
(n=268)\end{array}$ & $\begin{array}{l}\text { Titrate } \\
(\mathrm{n}=1,230)\end{array}$ \\
\hline \multicolumn{7}{|l|}{ BL LDL-C* } \\
\hline $\mathrm{mmol} / \mathrm{L}(\mathrm{SD})$ & $3.1(0.8)$ & $2.8(0.7)$ & $2.9(0.8)$ & $2.7(0.7)$ & $2.9(0.8)$ & $2.6(0.8)$ \\
\hline mg/dL (SD) & $118.2(29.5)$ & $108.2(27.8)$ & II 2.9 (30.5) & $103.8(28.5)$ & I I 2.7 (30.4) & $101.7(32.6)$ \\
\hline \multicolumn{7}{|l|}{ FW LDL-C* } \\
\hline $\mathrm{mmol} / \mathrm{L}(\mathrm{SD})$ & $2.2(0.7)$ & $2.5(0.7)$ & $2.1(0.7)$ & $2.3(0.7)$ & $2.1(0.7)$ & $2.3(0.7)$ \\
\hline $\mathrm{mg} / \mathrm{dL}(\mathrm{SD})$ & $84.8(28.0)$ & $94.9(25.8)$ & $80.3(25.6)$ & $90.5(25.2)$ & $80.3(27.9)$ & $88.6(28.7)$ \\
\hline \multicolumn{7}{|c|}{ Difference in LDL-C } \\
\hline FW versus & -33.4 & -13.3 & -32.6 & -13.3 & -32.3 & -19.3 \\
\hline BL* $(95 \% \mathrm{Cl})$ & $(-35.8,-31.0)$ & $(-14.1,-12.6)$ & $(-34.0,-3 \mid .2)$ & $(-13.9,-12.7)$ & $(-35.8,-28.9)$ & $(-14.8,-11.3)$ \\
\hline
\end{tabular}

Note: *Significant at $5 \%$ level for add-on versus titrate.

Abbreviations: BL, baseline; FW, follow-up; LDL-C, low-density lipoprotein cholesterol; SD, standard deviation; Cl, confidence interval. 


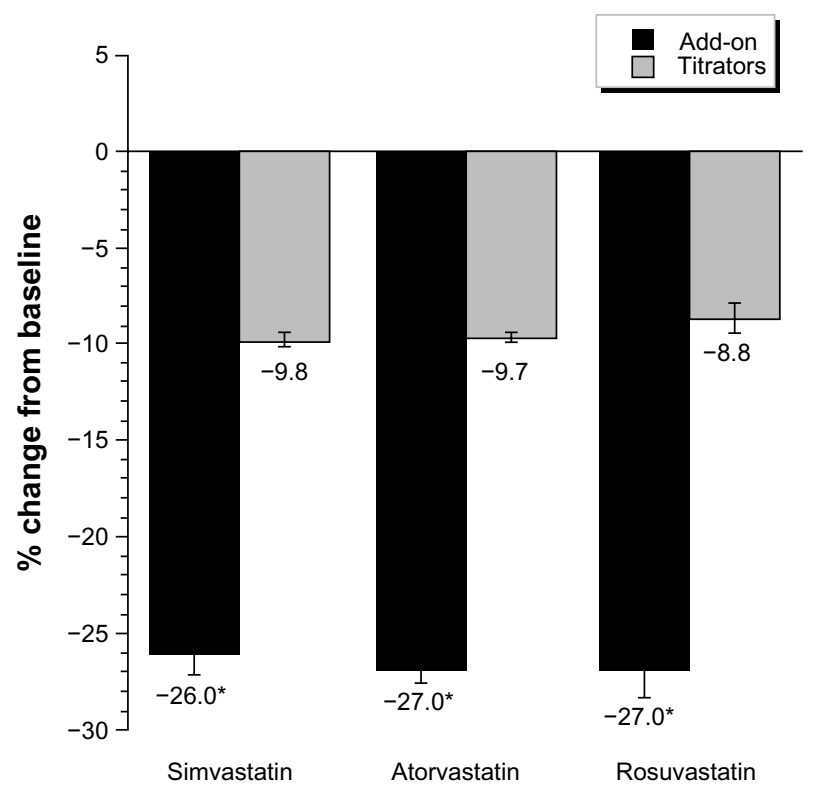

Figure 2 Percent change from baseline in LDL-C.

Note: $*$ Significant at $5 \%$ level for add-on versus titrate therapy.

Abbreviation: LDL-C, low-density lipoprotein cholesterol.

based on clinical trial data, many patients may not tolerate high doses required to achieve clinical targets. Combination therapy with ezetimibe plus statins has been shown to be safe and efficacious in numerous randomized, controlled clinical studies. ${ }^{10,27}$ In our study, ezetimibe added onto statin therapy resulted in greater percent LDL-C changes from baseline $(-26 \%$ to $-27 \%)$ compared with statin uptitration $(-8.8 \%$ to $-9.8 \%)$, consistent with the LDL-C reductions $(-21 \%$ to $-27 \%$ ) observed previously in clinical studies in which ezetimibe was added onto ongoing simvastatin, atorvastatin, or rosuvastatin monotherapy. ${ }^{10,27}$ Moreover, the significant additional 16\%-18\% LDL-C reduction observed in our study with ezetimibe add-on versus statin uptitration is also in line with clinical studies in which the addition of ezetimibe to statin therapy significantly reduced LDL-C by $14 \%-21 \%$ more than doubling the statin dose..$^{10,27}$

Multiple observational and randomized clinical studies have shown that statin uptitration and/or combination therapy with higher LDL-C-lowering efficacy is more effective than moderate-potency therapy for reducing LDL-C and improving goal attainment in high-risk patients. ${ }^{28-34}$ Our study also showed that treatment with higher-potency, LDL-C-lowering efficacy levels of ezetimibe add-on or statin uptitration therapies improved both LDL-C reductions and goal attainment, highlighting the potential benefit of more intensive therapy in reaching LDL-C goals in high-risk patients. Nonetheless, a substantial number of patients still did not attain LDL-C $<1.8 \mathrm{mmol} / \mathrm{L}(70 \mathrm{mg} / \mathrm{dL})$, as shown previously in studies of combination statin plus ezetimibe or niacin therapy, and statin uptitration, where $<50 \%$ of patients attained LDL-C $<1.8 \mathrm{mmol} / \mathrm{L}(70 \mathrm{mg} / \mathrm{dL}){ }^{7,35}$ These studies also reported that high-potency statin therapy and/or combination therapy were used less frequently in clinical practice compared with moderate-potency lipid-lowering therapy, likely due to intolerance or cost factors. Similarly, although the reasons for therapy choice were not specifically evaluated

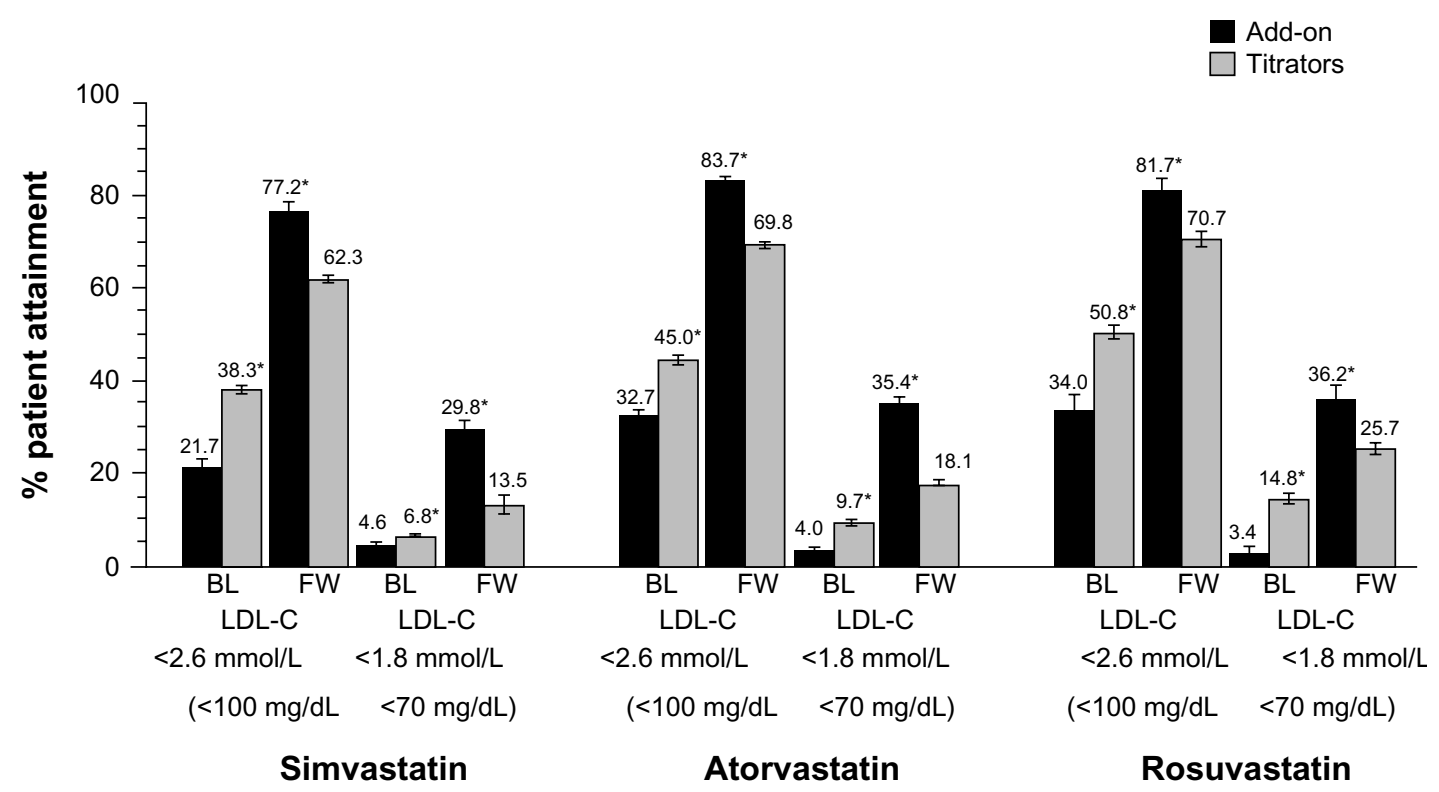

Figure 3 Percent attainment of LDL-C $<2.6$ and $1.8 \mathrm{mmol} / \mathrm{L}$ ( 100 and $70 \mathrm{mg} / \mathrm{dL}$ ).

Note: *Significant at $5 \%$ level for statin titration versus add-on baseline therapy and for add-on versus titrate follow-up therapy.

Abbreviations: BL, baseline; FW, follow-up; LDL-C, low-density lipoprotein cholesterol. 
Table 4 Multivariate models of percent change from baseline in LDL-C and the odds of goal attainment

\begin{tabular}{llll}
\hline Parameter & $\begin{array}{l}\text { Add ezetimibe versus } \\
\text { titrate simvastatin }\end{array}$ & $\begin{array}{l}\text { Add ezetimibe versus } \\
\text { titrate atorvastatin }\end{array}$ & $\begin{array}{l}\text { Add ezetimibe versus } \\
\text { titrate rosuvastatin }\end{array}$ \\
\hline Difference $(95 \% \mathrm{Cl})^{\dagger}$ & -13.1 & -14.8 & -14.4 \\
$\%$ change from baseline & $(-15.4,-10.8)$ & $(-16.3,-13.4)$ & $(-18.0,-10.7)$ \\
Odds $(95 \% \mathrm{Cl})^{\ddagger}$ attainment $\mathrm{FW}$ & 2.8 & 3.2 & 2.6 \\
$\mathrm{LDL}-\mathrm{C}<2.6 \mathrm{mmol} / \mathrm{L}(100 \mathrm{mg} / \mathrm{dL})$ & $(2.1-3.6)$ & $(2.7-3.9)$ & $(1.8-3.8)$ \\
Odds $(95 \% \mathrm{Cl})^{\ddagger}$ attainment $\mathrm{FW}$ & 2.9 & 3.1 & 2.5 \\
$\mathrm{LDL}-\mathrm{C}<1.8 \mathrm{mmol} / \mathrm{L}(70 \mathrm{mg} / \mathrm{dL})$ & $(2.2-3.8)$ & $(2.7-3.7)$ & $(1.8-3.4)$ \\
\hline
\end{tabular}

Notes: ${ }^{\dagger}$ Percent change from baseline in LDL-C was assessed by an analysis of covariance model adjusted for age, sex, baseline LDL-C, and baseline statin potency; ${ }^{\dagger}$ odds of attainment of LDL-C goals were assessed by logistic regression adjusted for age, sex, baseline goal attainment, and statin potency.

Abbreviations: $\mathrm{Cl}$, confidence interval; FW, follow-up; LDL-C, low-density lipoprotein cholesterol.

in our study, the number of patients on high-potency levels of statins was less than that on medium-potency statins, and overall use of ezetimibe add-on therapy was much lower than that of statin therapy. It should be noted that a lack of definitive data demonstrating the benefit of statin therapy in combination with ezetimibe on cardiovascular outcomes compared with statin monotherapy may limit its use, and an ongoing trial may provide additional information on its role in clinical practice. ${ }^{36}$

A strength of this study is the assessment of the effects of lipid-lowering therapy in a real-world clinical practice setting. However, our study has some limitations related to analyses in any retrospective, observational database. Due to a lack of specific information regarding all CHD risk factors (eg, smoking status) in the database that would distinguish those with a 10-year CHD risk greater than $20 \%$ as calculated by the Framingham Risk Score, it was not feasible to ascertain CHD risk-equivalent patients fully. Additionally, we did not have medical history for the time period preceding patient identification for this study, nor reasons for patients switching to add-on or uptitration therapies. Baseline data obtained in the database were also limited; thus, other factors such as concomitant medications and lifestyle changes (eg, weight loss, diet, exercise, comorbidity) that could affect LDL-C levels were also not assessed. Although the sample size of the groups was restricted somewhat by the availability of LDL-C values for only a subset of CHD/CHD risk-equivalent patients in the database, and the majority of the analysis population uptitrated statins compared with ezetimibe add-on therapy, the study still had adequate power to detect statistically significant differences in LDL-C levels and goal attainment. It is conceivable that variability in baseline and study-end LDL-C values, timing of followup analyses, as well as differences in baseline LDL-C values between the two treatment groups may have also affected the overall results; however, our findings are consistent with those reported in other studies. Finally, while our study indicates that ezetimibe added onto statin therapy improves LDL-C reduction and goal attainment in high-risk patients, our study was not designed to evaluate the effects of therapy on clinical outcomes; thus, further studies are needed.

In summary, our study in a real-world setting shows that addition of ezetimibe to ongoing simvastatin, atorvastatin, or rosuvastatin therapy improves LDL-C reduction and goal attainment in CHD/CHD risk-equivalent patients more than uptitration of these statins or switching to more potent statin monotherapies. Whether combination ezetimibe/ statin therapy is comparable with the use of high-potency statins in cardiovascular disease risk reduction remains to be determined. In the recent SHARP (Study of Heart and Renal Protection) trial, combination ezetimibe/simvastatin $(10 / 20 \mathrm{mg})$ reduced the incidence of major atherosclerotic events and was well tolerated in patients with chronic kidney disease during 4.9 years. ${ }^{37}$ Combination ezetimibe/simvastatin $(10 / 40 \mathrm{mg})$ was shown to reduce ischemic events in the SEAS (Simvastatin and Ezetimibe in Aortic Stenosis) study, consistent with expectations for the degree of LDL-C lowering achieved in patients with less severe aortic stenosis. ${ }^{1,38,39}$ While the SEAS and SHARP studies suggest that the LDL-C reductions produced by ezetimibe/simvastatin yield similar major cardiovascular event reductions as would be seen with the same magnitude of LDL-C lowering produced by statin monotherapy, ${ }^{37-39}$ the incremental benefit of combination ezetimibe and statin therapy compared with statin monotherapy is being addressed in the ongoing IMPROVEIT (Improved Reduction of Outcomes: Vytorin Efficacy International Trial) ${ }^{36}$

\section{Acknowledgment}

The authors thank Martha C Vollmer of Merck \& Co., Inc., Whitehouse Station, NJ, USA, for editorial assistance in the preparation of this manuscript. 


\section{Author contributions}

All authors jointly developed the manuscript content and were involved in at least one of the following: conception, design, data acquisition, analysis, statistical analysis, interpretation of data, drafting the manuscript and/or revising the manuscript for important intellectual content. All authors provided final approval of the version to be published.

\section{Disclosure}

JMF served as a consultant and/or member of an advisory board for Merck, Merck/Schering-Plough Pharmaceuticals, Pfizer, and Schering-Plough. PPT reports serving on speakers' bureaus for Abbott, AstraZeneca, Merck, and Kowa, and as a consultant for Amgen, Atherotech, Kowa, and Merck. HH, DN, DRR, SS, AMT, JET, and KT are employees of Merck and own stock/stock options in the company. This research was supported by Merck Sharp \& Dohme Corporation, Whitehouse Station, NJ, USA. The authors report no other conflicts of interest in this work.

\section{References}

1. Baigent C, Blackwell L, Emberson J, et al. Efficacy and safety of more intensive lowering of LDL cholesterol: a meta-analysis of data from 170,000 participants in 26 randomised trials. Lancet. 2010;376(9753): $1670-1681$.

2. Catapano AL, Reiner Z, De Backer G, et al. ESC/EAS Guidelines for the management of dyslipidaemias. The Task Force for the management of dyslipidaemias of the European Society of Cardiology (ESC) and the European Atherosclerosis Society (EAS). Atherosclerosis. 2011;217(1):3-46.

3. Grundy SM, Cleeman JI, Merz CN, et al. Implications of recent clinical trials for the National Cholesterol Education Program Adult Treatment Panel III Guidelines. J Am Coll Cardiol. 2004;44(3): 720-732.

4. Foody JM, Sajjan SG, Hu XH, et al. Loss of early gains in low-density lipoprotein cholesterol goal attainment among high-risk patients. J Clin Lipidol. 2010;4(2):126-132.

5. Nichols GA, Nag S, Chan W. Intensity of lipid-lowering therapy and low-density lipoprotein cholesterol goal attainment among the elderly before and after the 2004 National Cholesterol Education Program Adult Treatment Panel III update. Am Heart J. 2007;154(3):554-560.

6. Waters DD, Brotons C, Chiang CW, et al. Lipid treatment assessment project 2: a multinational survey to evaluate the proportion of patients achieving low-density lipoprotein cholesterol goals. Circulation. 2009;120(1):28-34

7. Kauffman AB, Olson KL, Youngblood ML, et al. Attainment of lowdensity lipoprotein cholesterol goals in coronary artery disease. J Clin Lipidol. 2010;4(3):173-180.

8. Kitkungvan D, Lynn Fillipon NM, Dani SS, Downey BC. Low-density lipoprotein cholesterol target achievement in patients at high risk for coronary heart disease. J Clin Lipidol. 2010;4(4):293-297.

9. Nag SS, Daniel GW, Bullano MF, et al. LDL-C goal attainment among patients newly diagnosed with coronary heart disease or diabetes in a commercial HMO. J Manag Care Pharm. 2007;13(8):652-663.

10. Catapano AL, Toth PP, Tomassini JE, Tershakovec AM. The efficacy and safety of ezetimibe co-administered with statin therapy in various patient groups. Clin Lipidol. 2012;8(1):13-41.
11. Toth PP, Ballantyne CM, Davidson MH et al. Changes in prescription patterns before and after reporting of the Ezetimibe and Simvastatin in Hypercholesterolemia Enhances Atherosclerosis Regression trial (ENHANCE) results and expected effects on low-density lipoproteincholesterol reduction. J Clin Lipidol. 2012;6(2):180-191.

12. Crestor (rosuvastatin calcium) tablets [package insert]. Wilmington, DE: AstraZeneca; 2013.

13. Lipitor (atorvastatin calcium) tablets [package insert]. New York, NY: Pfizer; 2013.

14. Zocor (simvastatin) tablets [package insert]. Whitehouse Station, NJ: Merck \& Co, Inc; 2012.

15. Liptruzet (ezetimibe/atorvastatin) [package insert]. Whitehouse Station, NJ: Merck \& Co, Inc; 2013

16. Vytorin (ezetimibe/simvastatin) [package insert]. Whitehouse Station, NJ: Merck \& Co, Inc; 2013.

17. Ballantyne CM, Blazing MA, King TR, Brady WE, Palmisano J. Efficacy and safety of ezetimibe co-administered with simvastatin compared with atorvastatin in adults with hypercholesterolemia. Am J Cardiol. 2004;93(12):1487-1494.

18. Ballantyne CM, Abate N, Yuan Z, King TR, Palmisano J. Dosecomparison study of the combination of ezetimibe and simvastatin (Vytorin) versus atorvastatin in patients with hypercholesterolemia: the Vytorin Versus Atorvastatin (VYVA) study. Am Heart J. 2005;149(3):464-473.

19. Weinstock RS, Goldberg RB, Guyton JR et al. Effect of ezetimibe/ simvastatin vs atorvastatin on lowering levels of LDL-C and non-HDL$\mathrm{C}, \mathrm{ApoB}$, and hs-CRP in patients with type 2 diabetes. J Clin Lipidol. 2008;2(1):25-35.

20. Bays HE, Ose L, Fraser N et al. A multicenter, randomized, double-blind, placebo-controlled, factorial design study to evaluate the lipid-altering efficacy and safety profile of the ezetimibe/simvastatin tablet compared with ezetimibe and simvastatin monotherapy in patients with primary hypercholesterolemia. Clin Ther. 2004;26(11):1758-1773.

21. Catapano AL, Davidson MH, Ballantyne CM et al. Lipid-altering efficacy of the ezetimibe/simvastatin single tablet versus rosuvastatin in hypercholesterolemic patients. Curr Med Res Opin. 2006;22(10):2041-2053.

22. Pearson TA, Denke MA, McBride PE et al. A community-based, randomized trial of ezetimibe added to statin therapy to attain NCEP ATP III goals for LDL cholesterol in hypercholesterolemic patients: the ezetimibe add-on to statin for effectiveness (EASE) trial. Mayo Clin Proc. 2005;80(5):587-595.

23. Kosoglou T, Statkevich P, Yang B et al. Pharmacodynamic interaction between ezetimibe and rosuvastatin. Curr Med Res Opin. 2004;20(8):1185-1195.

24. Ballantyne CM, Weiss R, Moccetti T et al. Efficacy and safety of rosuvastatin $40 \mathrm{mg}$ alone or in combination with ezetimibe in patients at high risk of cardiovascular disease (results from the EXPLORER study). Am J Cardiol. 2007;99(5):673-680.

25. Steinberg BA, Bhatt DL, Mehta S, et al. Nine-year trends in achievement of risk factor goals in the US and European outpatients with cardiovascular disease. Am Heart J. 2008;156(4):719-727.

26. Vande Griend JP, Saseen JJ. Low-density lipoprotein cholesterol goal attainment in high-risk family medicine patients. J Clin Lipidol. 2009;3(3):195-200.

27. Toth PP, Catapano A, Tomassini JE, Tershakovec AM. Update on the efficacy and safety of combination ezetimibe plus statin therapy. Clin Lipidol. 2010;5(5):655-684.

28. Aronow HD, Hess G, Hill J, Kuznik A, Liu LZ. Switching from atorvastatin to simvastatin in patients at high cardiovascular risk: effects on low-density lipoprotein cholesterol. Am J Ther. 2010;17(2): $167-175$.

29. Atar D, Carmena R, Clemmensen P, et al. Clinical review: impact of statin substitution policies on patient outcomes. Ann Med. 2009;41(4): 242-256. 
30. Athyros VG, Tziomalos K, Karagiannis A, Mikhailidis DP. To switch (statins) or not to switch? That is the question. Expert Opin Pharmacother. 2010;11(18):2943-2946.

31. Jones PH, Davidson MH, Stein EA, et al. Comparison of the efficacy and safety of rosuvastatin versus atorvastatin, simvastatin, and pravastatin across doses (STELLAR* Trial). Am J Cardiol. 2003;92(2):152-160.

32. Mikhailidis DP, Sibbring GC, Ballantyne CM, Davies GM, Catapano AL. Meta-analysis of the cholesterol-lowering effect of ezetimibe added to ongoing statin therapy. Curr Med Res Opin. 2007;23(8):2009-2026.

33. Tunceli K, Sajjan SG, Ramey DR, et al. Switching from high-efficacy lipid-lowering therapies to simvastatin and low-density lipoprotein cholesterol goal attainment in coronary heart disease/coronary heart disease-equivalent patients. J Clin Lipidol. 2010;4(6):491-500.

34. Willey VJ, Bullano MF, Shoetan NN, Gandhi SK. Therapy modifications and low-density lipoprotein cholesterol goal attainment rates associated with the initiation of generic simvastatin. Curr Med Res Opin. 2010;26(1):121-128.
35. Karalis DG, Victor B, Ahedor L, Liu L. Use of lipid-lowering medications and the likelihood of achieving optimal LDL-cholesterol goals in coronary artery disease patients. Cholesterol. 2012;2012:861924.

36. Califf RM, Lokhnygina Y, Cannon CP, et al. An update on the IMProved reduction of outcomes: Vytorin Efficacy International Trial (IMPROVEIT) design. Am Heart J. 2010;159(5):705-709.

37. Baigent $\mathrm{C}$, Landray MJ, Reith $\mathrm{C}$, et al. The effects of lowering LDL cholesterol with simvastatin plus ezetimibe in patients with chronic kidney disease (Study of Heart and Renal Protection): a randomised placebo-controlled trial. Lancet. 2011;377(9784):2181-2192.

38. Holme I, Boman K, Brudi P, et al. Observed and predicted reduction of ischemic cardiovascular events in the Simvastatin and Ezetimibe in Aortic Stenosis trial. Am J Cardiol. 2010;105(12):1802-1808.

39. Rossebo AB, Pedersen TR, Boman K, et al. Intensive lipid lowering with simvastatin and ezetimibe in aortic stenosis. $N$ Engl J Med. 2008;359(13):1343-1356.
Vascular Health and Risk Management

\section{Publish your work in this journal}

Vascular Health and Risk Management is an international, peerreviewed journal of therapeutics and risk management, focusing on concise rapid reporting of clinical studies on the processes involved in the maintenance of vascular health; the monitoring, prevention and treatment of vascular disease and its sequelae; and the involvement of

\section{Dovepress}

metabolic disorders, particularly diabetes. This journal is indexed on PubMed Central and MedLine. The manuscript management system is completely online and includes a very quick and fair peer-review system, which is all easy to use. Visit http://www.dovepress.com/ testimonials.php to read real quotes from published authors.

Submit your manuscript here: http://www.dovepress.com/vascular-health-and-risk-management-journal 